\title{
FFAG LATTICE FOR MUON ACCELERATION WITH DISTRIBUTED RF*
}

\author{
D. Trbojevic, J.S. Berg, M.Blaskiewicz, E.D. Courant, R. Palmer, BNL, Upton, New York \\ A. Garren, LBL, Berkeley, California, USA
}

\begin{abstract}
A future muon collider or neutrino factory requires fast acceleration to minimize muon decay. We have previously described an FFAG ring that accelerated muons from 10 to $20 \mathrm{GeV}$ in energy. The ring achieved its large momentum acceptance using a low-emittance lattice with a small dispersion. In this paper, we present an update on that ring. We have used design tools that more accurately represent the ring's behavior at large momentum offsets. We have also improved the dynamic aperture from the earlier design.
\end{abstract}

\section{INTRODUCTION}

Fixed field alternating gradient (FFAG) lattice designs [1] have been extensively used and studied in recent years [2]. Fast acceleration of large emittance muon beams, is essential for either neutrino factory or muon collider projects because of the short muon lifetime. The KEK feasibility study in 2001 had chosen the standard FFAG lattice design for the muon acceleration and "cooling". In addition, a small "proof of principle" $50-500 \mathrm{KeV}$ FFAG proton ring was built and commissioned [2]. The advantages of this design are: the large momentum acceptance, fixed magnetic field during acceleration, relatively large energy gain per turn, transition energy above the output energy, etc. The standard design is a socalled "scaling" synchrotron where the betatron tunes are constant during acceleration, giving zero chromaticity. Particles at different energies follow orbits parallel to each other during acceleration. A disadvantage of this "scaling" FFAG designs is that it has a large required aperture with a large circumference ratio due to required negative bending. The present report follows the basic principle of the FFAG described by Symon [1] but with a non-scaling FFAG using the minimum emittance lattice design [3]. The disadvantages of the scaling FFAG design are dramatically reduced. A relatively small FFAG synchrotron with a circumference of $\sim 300 \mathrm{~m}$ can be designed to accelerate muons from energy of $10 \mathrm{GeV}$ up to $20 \mathrm{GeV}$ in about $\sim 20$ turns. It is assumed that the magnets and the RF are super-conducting.

\section{Organization of the Report:}

The next section presents the basic idea of the design. The lattice properties calculated at the central momentum of $E_{o}=15 \mathrm{GeV}$ are described in the third section. The lattice function dependence on momentum is described in the fourth section (the amplitude functions, tunes, orbits, momentum compaction, circumference). The acceleration is described in the fifth and the section 6 is aconclusion.

\section{LATTICE PROPERTIES OF A CELL}

The basic idea of accelerating muons with the FFAG scaling synchrotron using the minimum emittance lattice has been described in detail earlier. To allow a large momentum acceptance and large energy change a small dispersion minimum emittance lattice is employed. The amplitude of the normalized dispersion function is defined as the square root of the dispersion emittance function $H$ [3]:

$$
H=\xi^{2}+\zeta^{2}, \quad \xi=\eta^{\prime} \sqrt{\beta}+\frac{\alpha \cdot \eta}{\sqrt{\beta}}, \zeta=\frac{\eta}{\sqrt{\beta}} .
$$

where $\eta$ is the dispersion function. The minimum emittance lattice is one that minimizes the function $H$ [3]. This is achieved having the minimum of the horizontal amplitude $\beta_{x}$ and dispersion function $\eta_{x}$ occur at the center of the bending element. If the bending element is a combined function magnet, the conditions are more favorable. This update of the previous designs includes three major simplifications: the necessary space for the accelerating cavities has been enlarged by removal of one of the magnets, and the basic cell betatron tune changes during acceleration are kept between half integer values, permitting the removal of sextupole magnets. A small opposite bend has been added to the focusing quadrupole; this improves the tune acceptance over the large momentum range of the basic cell.

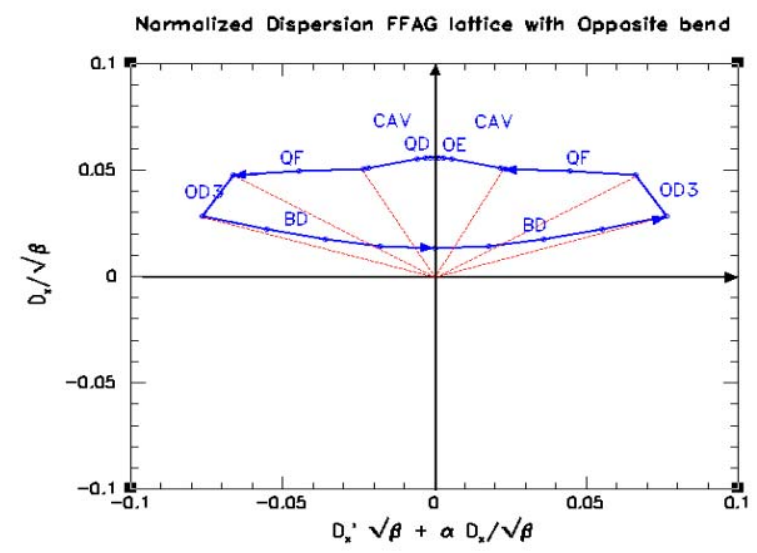

Figure 1: Normalized dispersion space: the opposite bend at $\mathrm{QF}$ quadrupole, while the major bend is at $\mathrm{BD}$.

The square root of the $\mathrm{H}$ function is presented by the red lines in Fig. 1. The opposite bend is in the focusing quadrupole labeled as QF. The normalized dispersion

*Work performed under Contract Number DE-AC02-98CH10886 with the auspicies of the US Deparment of Energy. 
vector changes for a $D^{\prime} \sqrt{ } \beta_{x}=\theta \sqrt{ } \beta_{x}$ at the bending elements. Due to the larger betatron function $\beta_{x}$ at the focusing quadrupole, the vector $\theta_{Q F} \sqrt{ } \beta_{x_{Q} Q F}$ is almost the same size as the one for the major bend $\theta_{B D} \sqrt{ } \beta_{x_{B} B D}$. This reduces the overall value of the $H$ function and allows large momentum acceptance. In Fig. 1 a drift, space or non-bending element is presented as a part of a circle. The circumference of the machine is $C \sim 300 \mathrm{~m}$; it consists of sixty identical cells $\sim 5$ meters long.

\section{THE BASIC CELL}

The basic FFAG minimum emittance cell is made of only two type of magnets: the central bending element is a combined function magnet, $l_{d} \sim 1.6 \mathrm{~m}$ long, with a defocusing gradient; the two focusing quadrupoles, $l_{Q F^{\sim}} \sim 0.55 \mathrm{~m}$ long, have equal but opposite focusing gradients and a negative bending field. As in the previous design the minimum of the horizontal betatron and dispersion functions are at the center of the dipole as presented in Fig. 2.

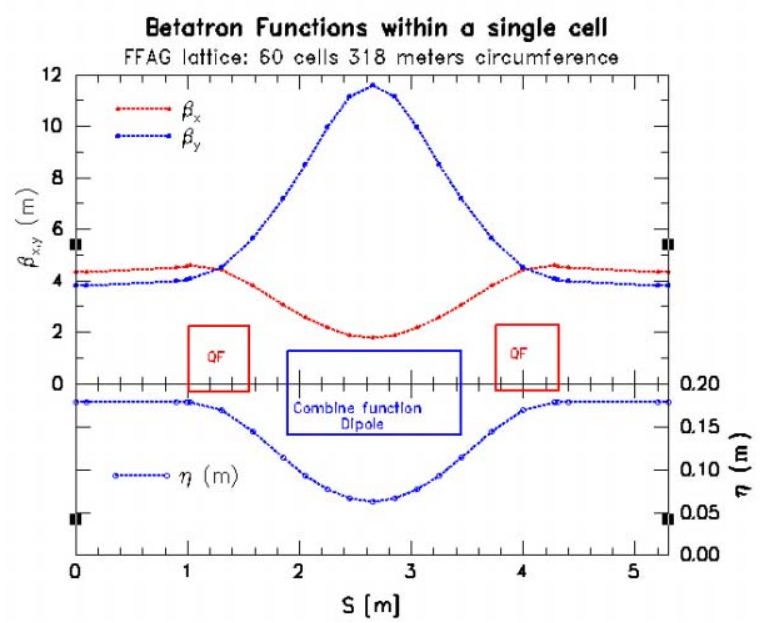

Figure 2: The betatron functions in the basic cell. The dispersion function is labeled as $\eta$.

The energy range for muon acceleration considered is between $10-20 \mathrm{GeV}$. The lattice functions are presented at the central energy equal to $E_{\mathrm{c}}=15 \mathrm{GeV}$. The lattice design tool used for this example is SYNCH [4]. Because it has been found [6] that other lattice programs [MAD, TEAPOT, COSY] have limitations at large momentum deviations. The betatron functions are calculated for the required momentum range of $\delta p / p= \pm 33 \%$. The magnets have sextupole strengths of $1 / 4$ of the strength that would make the chromaticity zero at the central momentum (stronger sextupoles would entail an unacceptably small dynamic aperture). A comparison of the calculations of the lattice function dependence on momentum for large momentum offsets obtained by four different lattice tools is presented at this conference [6]. The betatron functions (closed orbit offsets $x_{c o}, \beta_{x}, \beta_{y}, v_{x}$, and $v_{y}$ ) are presented without $1 / 4$ sextupoles strength, while they were used to study the influence on the orbit circumference and in longitudinal tracking.

\section{BETATRON FUNCTIONS DEPENDENCE ON MOMENTUM}

The incorporation of opposite bend in the focusing quadrupole allows tune changes during acceleration within the basic cell to be within a range which avoids integer and half integer resonances $\left(0.1<v_{x, y}<0.4\right)$. This is a necessary condition for a stable particle motion. The dependence of the tunes on momentum within the basic cell is shown in Fig. 3.

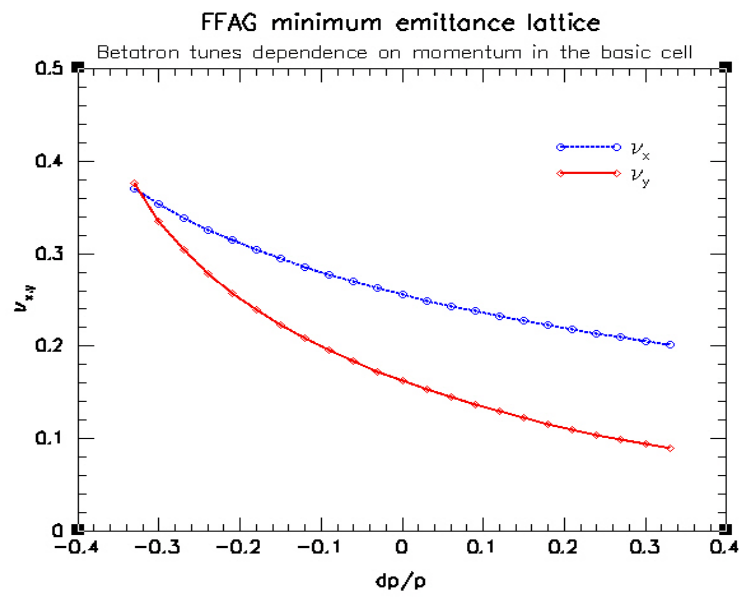

Figure 3: Betatron tunes, $v_{x}$ and $v_{y}$, dependence on a large range of momentum in the basic cell.

The betatron functions $\beta_{x}$ and $\beta_{y}$ should have moderate values over the required momentum range. When the vertical tune comes close to an integer or half-integer the maximum of the vertical betatron function becomes larger, as shown in Fig 4.

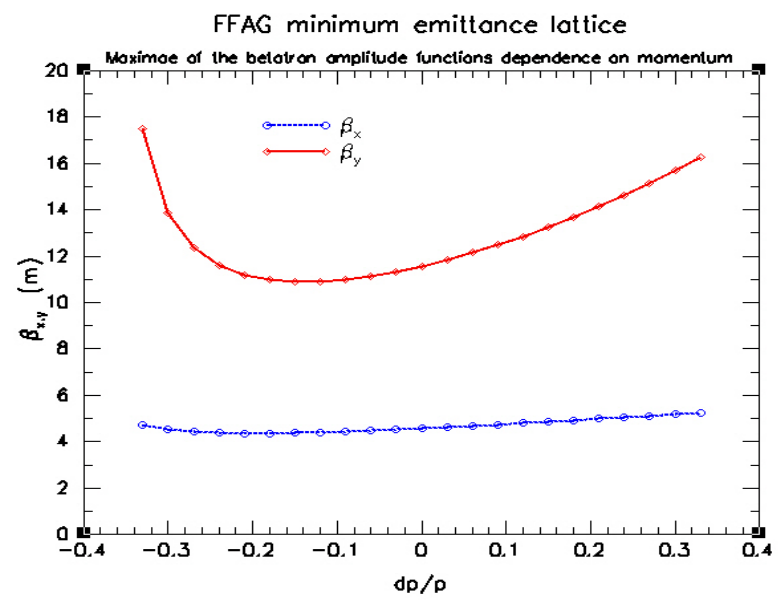

Figure 4: Betatron functions dependence on momentum.

The particle orbits during acceleration through a single cell are the most important aspect of this lattice design, as shown in Fig. 5. Some important details from this figure need to be emphasized: at the start of acceleration particles move through the basic cell on the inside of the aperture and have mostly negative closed orbit offsets $\left(\mathrm{x}_{\mathrm{co}}<0\right)$. The largest orbit offsets at the lower energy side in this example are of the order of $x_{c o} \sim 50 \mathrm{~mm}$. As the particles are accelerated from $E_{o}=10 \mathrm{GeV}$ up to $E_{m}=20$ 
$\mathrm{GeV}$, the orbits move towards the outside of the magnets' aperture with a zero closed orbit for the central energy of $E_{c}=15 \mathrm{GeV}$. The closed orbit offsets at the maximum energy reach values of $x_{c o} \sim 58 \mathrm{~mm}$. These closed orbit offsets may be compared to offsets of the order of $x_{c o} \sim 500$ $\mathrm{mm}$, for the same energy range $10-20 \mathrm{GeV}$, in the scaling FFAG [2].

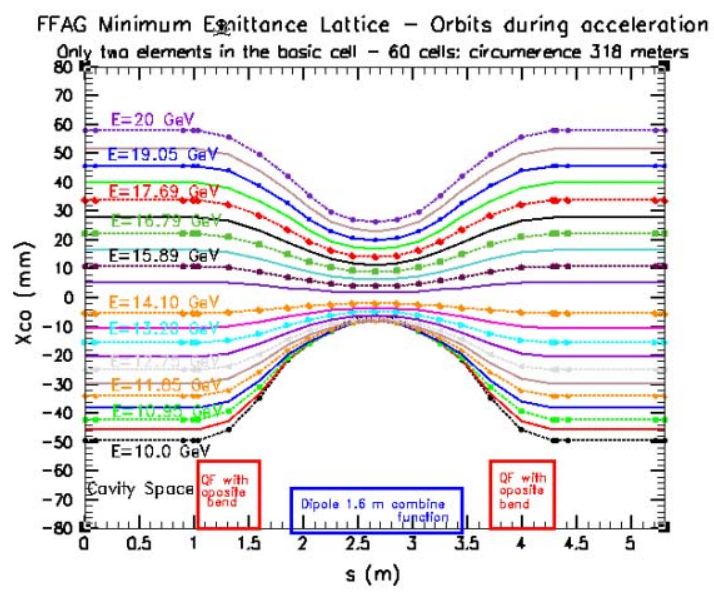

Figure 5: Closed orbit offsets during acceleration from the muon energy of $10 \mathrm{GeV}$ to $20 \mathrm{GeV}$, in the basic cell.

\section{Acceleration Parameters: $\alpha_{c}$ - the Momentum} Compaction and a Difference in the Path Length

The dependence of the momentum compaction $\alpha_{c}$ on energy is approximately linear. At the lowest energy the lattice has imaginary $\gamma_{t}$, and as the energy crosses the central energy $\gamma_{t}$ becomes real. The path length difference during acceleration, as calculated by $\mathrm{SYNCH}$, is shown in Fig. 6.

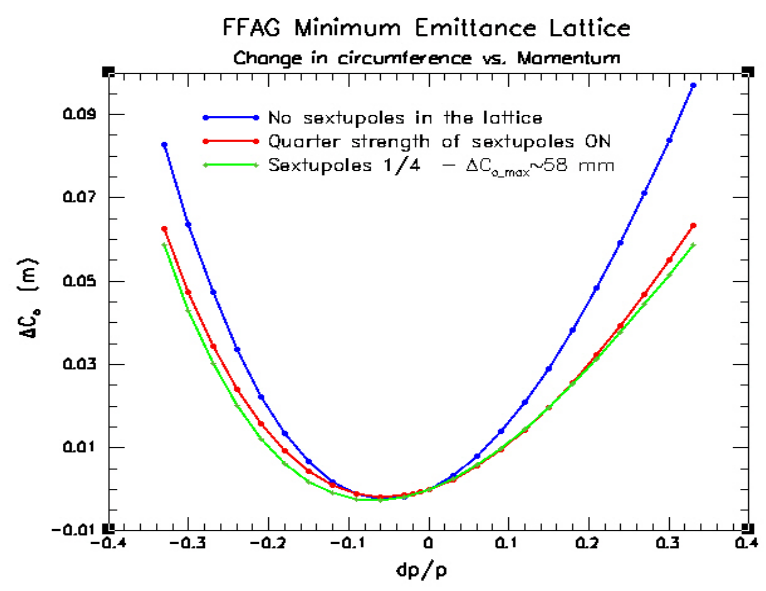

Figure 6: Change in circumference during acceleration.

\section{ACCELERATION}

A simulation of the longitudinal motion as presented in Fig. 7, was performed with the assumption that the path difference is the same as presented in Fig. 6 with quarter strength of sextupoles. More details about this simulation have been previously presented [7]. Parameters used for the simulation are: 10 bunches, $0.1 \mu C /$ bunch, initial bunch half length $1.6 \mathrm{~ns}$, initial half energy spread $4 \mathrm{MeV}$, initial $200 \mathrm{MHz}$ RF voltage $650 \mathrm{MV} / \mathrm{turn}$, final vs. initial emittance $=1.125$, and a for the beam loading a fraction of stored cavity energy absorbed by the beam, $45 \%$.

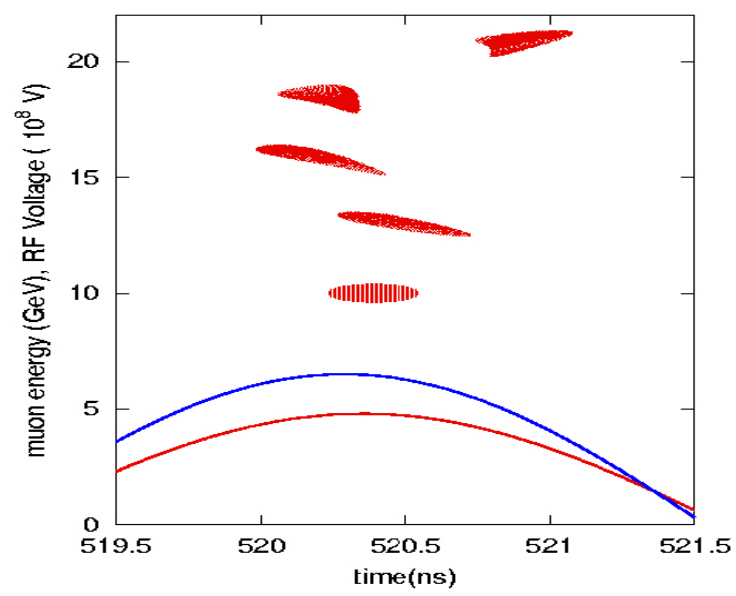

Figure 7: Longitudinal simulation of acceleration.

\section{CONCLUSIONS}

We have described an example of the non-scaling FFAG lattice and its advantages with respect to the standard scaling FFAG concept. When compared with the scaling examples the aperture size is significantly smaller as well as the circumference. The super-conducting combined function magnets are within a reasonable size and strength. The muon acceleration, the major cost item in the neutrino factory feasibility studies, is becoming more realistic and less costly.

\section{REFERENCES}

[1] K. R. Symon, “The FFAG Synchrotron - MARK I”, MURAKRS-6, November 12, 1954, pp. 1-19.

[2] R. Ueno et al, "Multi Orbit Synchrotron with FFAG Focusing For Acceleration of High Intensities Hadron Beams", PAC 99, New York, 1999, 2271-2273.

[3] D. Trbojevic and D. Courant, E., "Low emittance lattices for electron storage rings revisited", 4th European Particle Accelerator Conference, London, England, June 27-July 1, 1994, pp. 1000-1002.

[4] A. Garren, A. S. Kenney, E. D. Courant, A. D. Russell, and M. J. Syphers, SYNCH-A Program for Design and Analysis of Synchrotrons and Beam Lines, User's Guide 1993.

[5] F.C. Iselin and H. Grote, MAD, "Methodical Accelerator Design”, CERN/SL, 90-13 (12991).

[6] E.D. Courant, J. S. Berg, A. Garren, R. Talman, and D. Trbojevic, "A Comparison of several lattice tools for Computation of orbit functions of an accelerator", this conference, EPAG010.

[7] D. Trbojevic, M. Blaskiewicz, E. D. Courant, and A. Garren, "Fixed field alternating gradient lattice design without opposite bend", Proceedings of EPAC2002, Paris, France,pp.1199-1201. 